\title{
A One-Motor Full-Mobility 6-PUS Manipulator
}

\author{
Patrick Grosch $^{\left({ }^{\circ}\right)}$, Raffaele Di Gregorio ${ }^{\left({ }^{\circ}\right)}$, Federico Thomas ${ }^{\left({ }^{\circ} *\right.}$ \\ $\left(^{\circ}\right.$ )Institut de Robòtica i Informàtica Industrial (CSIC-UPC), Barcelona, Spain \\ $\left({ }^{\circ}\right)$ Department of Engineering - University of Ferrara, Ferrara, Italy
}

\begin{abstract}
This paper presents the feasibility study of an under-actuated parallel manipulator with 6-PUS topology, destined to handle work-tables in CNC machine tools. The proposed device exploits the fact that, in such an application, the path between the initial and final poses of the mobile platform is not assigned to reduce the number of actuators to only one.
\end{abstract}

\section{Introduction}

The need of making an object move along an assigned path arises only in a limited number of applications. In most cases, the only initial and final poses of the object are assigned, whereas the path between them must just satisfy weak constraints (e.g., obstacle avoidance, preventing interferences among machine components, etc.) which leave the choice of the path practically free. Such a freedom can be exploited during design to simplify the machine architecture.

Work-tables of machine tools usually either perform simple translations or just lock the workpiece during cutting. Thus every time the workpiece has to be reoriented or, in general, repositioned with respect to the spindle axis either manual operations or external devices must intervene. Repositioning workpieces is a manipulation task that involves small six-dimensional workspaces, good positioning precision and high stiffness in the final configuration; it does not impose any constraint to the path between the initial and final poses.

Parallel manipulators can satisfy the requirements on positioning precision and stiffness; moreover, they are specially suitable for applications that involve small workspaces. Therefore, they are natural candidates to move the work-table during workpiece repositioning.

\footnotetext{
* This work was supported by the Spanish Ministry of Science and Innovation, by the contribution of Regione Emilia Romagna (District Councillorship for Productive Assets, Economic Development, Telematic Plan), PRRIITT misura 3.4 azione A, to InterMech (Division Acoustics and Vibrations - LAV), and by UNIFE funds.
} 
How to exploit the free path for reducing the complexity of a manipulator destined to move the work-table during repositioning is an open problem.

This paper proposes an under-actuated parallel manipulator that, by exploiting the free path, is able to control the mobile platform pose in a six-dimensional workspace by using only one motor. Section 2 describes the manipulator architecture and illustrates its operation. Section 3 addresses the kinetostatic analysis of the machine and gives conditions the path must satisfy to keep the mobile platform pose controllable during motion. Eventually, the conclusions are drawn in section 4.

\section{Manipulator Architecture and Operation}

Parallel manipulators (PMs) with topology 6-PUS ${ }^{1}$ feature a mobile platform connected to a fixed base through six in-parallel kinematic chains (legs) of type PUS. Their architectures vary according to the relative disposition of the prismatic-pair sliding directions, the platform geometry, and the six fixed distances (leg lengths) between universal-joint center and spherical-pair center of each leg. By changing these geometric parameters, a number of 6-PUS PMs have been proposed in the literature (see Merlet (2006, chap. 2) for Refs.). Boye and Pritschow (2005) named them linapods. Honegger et al. (1997) proposed the Hexaglide that has six parallel and coplanar guides. Moreover, some of the proposed architectures (Bernier et al. 1995; Pritschow et al. 2002) exhibit coincident guides for couples of prismatic pairs, and, in particular, Nabla 6, proposed by Bernier et al. (1995), has three coplanar guides each carrying two sliders. The actuation of each prismatic pair is independent of the other actuations in all the linapods proposed in the literature.

Figure 1 shows the proposed linapod. On the base, a single motor, through a transmission, actuates, one at a time, two racks that are constrained to slide along two mutually orthogonal guides forming a cross-shaped path. The transmission is able to actuate one or the other rack by using two clutches that also act as brakes for the non-actuated rack. The racks carry suitably shaped hooks which can firmly lock revolute-pair housings (the cubes attached to the racks in Fig. 1). In these revolute-pair housings, legs' universal joints insert one pin of their cross link so that the resulting revolute pair has the axis perpendicular to the plane of the guides. In so doing, all the universal joints have the other revolute-pair axis parallel to the plane of the guides, and their centers are constrained to lie on $\mathrm{T}$ paths that are all parallel to the plane of the guides. The universal-joint centers slide on these $\mathrm{T}$ paths when the racks are moved.

On the mobile platform, the housing of the spherical pairs, which join the leg endings to the platform, are embedded in the platform.

${ }^{1} \mathrm{P}, \mathrm{U}$, and $\mathrm{S}$ stand for prismatic pair, universal joint, and spherical pair, respectively; whereas, the underscoring indicates that the corresponding joint is actuated. 


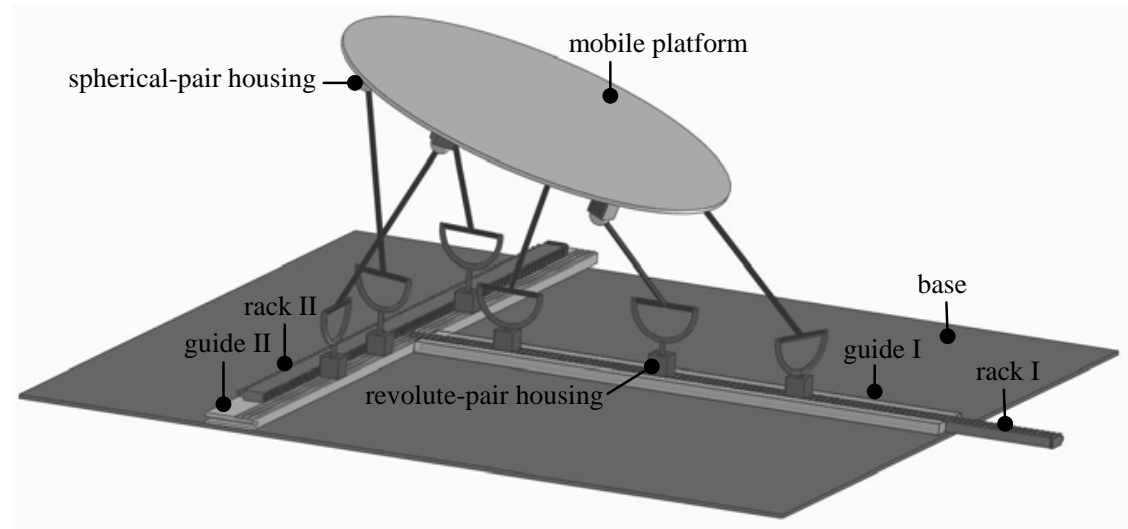

(a)

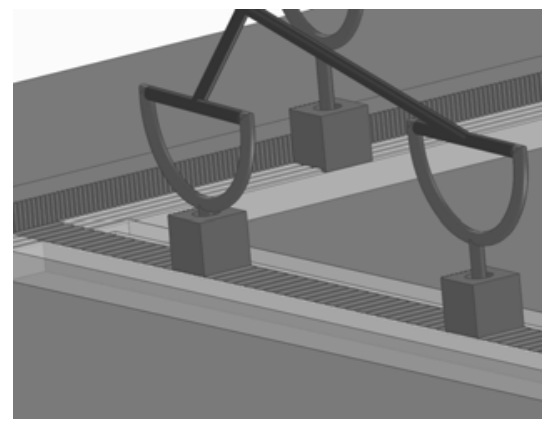

(b)

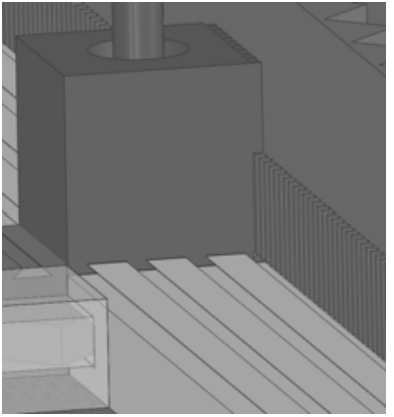

(c)

Figure 1. Under-actuated 6-PUS: (a) 3D CAD model, (b) detail of the racks, the guides, and some of the revolute-pair housings, (c) revolute-pair housing at the intersection between the two racks.

The hooking between rack and revolute-pair housing is managed by a purely mechanical device carried either on the revolute-pair housing or on the guides. This hooking device and the hooks on the racks are conceived so that the following functional requirements are satisfied: 
(i) if the revolute-pair housing is in contact with only one rack, the hooking device must provide a constraint that, combined with the constraint of rack's hooks, firmly holds the housing fixed to the rack;

(ii) if the revolute-pair housing is in contact with both racks (i.e., at the intersection of the T path), the hooking device must not provide any constraint, whereas the hooks of both racks must provide the constraints necessary to hold the housing;

(iii) if the revolute-pair housing is at the intersection of the $\mathrm{T}$ path and one rack starts moving, the hooks of the moving rack must be able to tow the housing, whereas the hooks of the other rack must not forbid this towing and must be so shaped that, during the housing motion, make the hooking device intervene to provide its constraint.
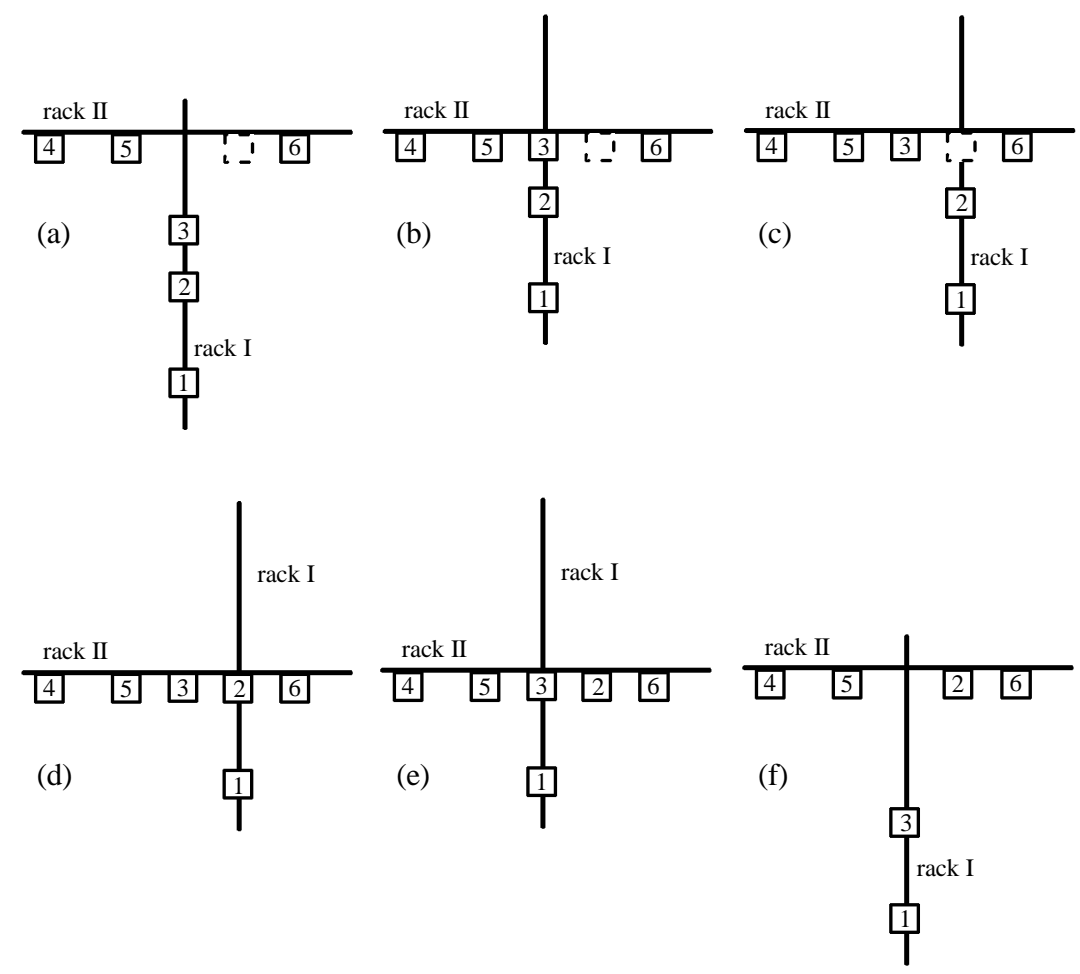

Figure 2. Sequence of rack motions (the numbered boxes represent the revolute-pair housings): (a) initial configuration, (b) rack I was moved to make housing 3 touch rack II, (c) rack II was moved to make the dotted box centered on rack I, (d) rack I was moved to make housing 2 touch rack II, (e) rack II was moved back to its initial position, (f) final configuration obtained by moving back rack I to its initial position. 
Many hooking devices and complementary hooks for the racks can be easily devised to satisfy the above technical requirements. For instance, in Fig. 1, the hooks of rack I are dovetail joints parallel to rack II, whereas the hooks of rack II are frontal teeth with rectangular cross section; moreover, the hooking device is constituted of two lateral stops fixed to guide I, and of dovetail joints, identical to the ones of rack I, fixed to guide II (see Figs. 1(b) and 1(c)). The many design alternatives for these equipments will not be discussed here, for the sake of conciseness.

By exploiting the above-reported properties of hooking device and racks' hooks the positions of the universal-joint centers on the $\mathrm{T}$ paths can be about freely changed. In fact, if, for instance, housing 2, on rack I, (Fig. 2(a)) must be moved to the dotted position on rack II and housing 3 must be moved to the actual position of housing 2, the sequence of operations shown in Fig. 2 can be implemented.

In general, many different rack-motion sequences lead to the same final configuration, and the number of operations to implement may decrease when the number of housing permutations increases.

\section{Kinetostatics and Constraints on the Path}

The inverse position analysis (IPA) that, for this linapod, means the determination of the housing positions on the racks for an assigned platform pose (position and orientation) must be solved every time the platform is repositioned. This determination is straightforward once the positions of the universal-joint centers have been computed. The assigned relative pose between base and platform involves that the six T paths (one per leg), the universal-joint centers must lie on, have assigned poses with respect to the spherical-pair centers embedded in the platform. Thus, for each leg, the determination of the universal-joint center's position reduces itself to compute the intersection points between the $\mathrm{T}$ path the universal-joint center must lie on and a sphere, with center at the spherical-pair center and radius equal to the leg length. This geometric problem has at most four solutions: the two sets of intersections between the sphere and the two sides of the T. At most four solutions for each leg yields at most $4^{6}$ (i.e., 4096) leg arrangements compatible with an assigned platform pose. Such a high number of IPA solutions is mainly theoretical. In fact, many line-sphere intersections will fall out of the line segments actually occupied by the T-path's sides. Moreover, other solutions will be excluded by the fact that two or more housings cannot be located on the same position, and that, on each rack, the hooks' sequence has a fixed pitch, which implies that the distance between couples of housings positioned on the same rack can only be multiples of the hooks' pitch. Eventually, all the leg arrangements that give a singular configuration (see below) must be excluded.

The direct position analysis (DPA) of the proposed linapod consists in the determination of the platform poses compatible with an assigned disposition of the 
revolute-pair housings on the two racks. If the positions of the revolute-pair housings are assigned, the positions of the universal-joint centers will be assigned, too. Thus this problem reduces itself to the determination of the assembly modes of the 6-US structure (i.e., two rigid bodies connected by six in-parallel US legs), which was broadly treated in the last two decades in connection with the DPA solution of the general Stewart platform (see Merlet (2006) for Refs.). The result of these studies is that the 6-US structure can have at most forty assembly modes which can be even analytically determined (Husty, 1996; Innocenti, 2001).

The singularities of the forward instantaneous kinematics are, for this linapod, the configurations where the platform can perform instantaneous motions even though the racks are locked (i.e., they are uncertainty configurations of the 6-US structure). At a singularity of this type, the platform pose is not controllable, and the internal loads of one or more links of the legs are not able to equilibrate the external loads applied on the platform. Thus, they must be identified during design and avoided during operation. The uncertainty configurations of the 6-US structure have been studied by many authors, and, in the literature, both geometric and analytic conditions to identify them have been provided (see, for instance, Merlet (1989), McCarthy (2000), St-Onge and Gosselin (2000), Di Gregorio (2002)). The actual implementation of the proposed linapod requires that all this literature be exploited to correctly design and control it. In this paper, for the sake of brevity, the authors will only give the justification of some design choices due to the need of avoiding uncertainty configurations.

From a static point of view, in 6-US structures, an uncertainty configuration occurs when the six forces applied to the platform through the spherical pairs are not able to equilibrate any system of external loads. The fact that these forces are aligned with the leg axes ${ }^{2}$ allowed the geometric classification of the singular configurations through particular arrangements of the six leg axes (McCarthy, 2000). All these singular arrangements satisfy at least one out of the following three geometric conditions: (a) the six axes either intersect or are parallel to a line, (b) the six axes are all parallel to a plane, and (c) the six axes are tangent to coaxial helices with the same pitch.

For the linapod under study, the possibility of locating all the revolute-pair housings on one rack would greatly improve the path planning algorithms (see below). Therefore, making this housing arrangement non-singular is important. Once all the housings are located on the same rack all the universal-joint centers lie on the same plane. And, in order to avoid the geometric conditions (a) and (b), the universal-joint centers must not be located at the same height on the rack (i.e., the $\mathrm{T}$ paths must not coincide); whereas the spherical-pair centers must be suitably dis-

${ }^{2}$ In a US leg, the leg axis is the line passing through the centers of the universal joint and of the spherical pair. 
tributed on the platform. The manufacturing conditions that allow condition (c) to be avoided are much more difficult to be visualized and a careful numerical check is necessary. It is worth noting that the leg arrangements with all the housings on the same rack geometrically coincide with the Hexaglide architecture (Honegger et al., 1997), and the results obtained for the Hexaglide can also be usefully exploited.

A path-planning algorithm for the proposed linapod has to take into account all the above-reported kinetostatic considerations. In addition, it needs the implementation of a motion strategy for choosing the sequence of rack motions able to move from the initial platform pose to the final one. Each step of this sequence finishes with a particular arrangement (state) of the revolute-pair housings on the racks that is reached when both the racks are at rest and the actuation is about to be switched from one rack to the other. Thus, a path-planning algorithm has to determine the states' sequence by respecting the rule that the transition from one state to the successive one must be possible by moving only one rack. For instance, the motion described in Fig. 2 is characterized by six states and five transitions. Two different paths that have the same initial and final housing arrangements can be compared on the basis of the number of intermediate states, and, of course, the lower is the number the better is the path.

The sequence that moves only one housing from any position to any other without changing the positions of the other housings, in the final state, can be easily automated. Thus, a simple path-planning algorithm could reduce itself to implement six separate sequences each of which brings only one housing from its initial to its final position and, in the final state, does not change the positions of the housings already brought to their final positions. Such a motion strategy employs a great number of intermediate states. For instance, it is easy to realize that, in Fig. 2, the motion of the only housing 2 without changing the position of housing 3 would have required nine states, whereas the strategy reported in Fig 2 uses only six states to move both housings 2 and 3 to their final positions.

A much better motion strategy can be obtained by finding a state (parking state) from which any other state can be reached through a reduced number of intermediate states. The state with all the housings located on rack II and no housing at the rack intersection could be a parking state. In fact, from this parking state, a housing can be put on rack I, at any position, with a sequence involving only two intermediate states, whereas only four intermediate states are required to change the position of a housing on rack II. A path-planning algorithm based on this parking state, first, has to implement the sequences that bring all the housings on rack II (note that the only housings located on rack I in the initial state are involved in this phase); then, it has to move all the housings from the parking positions to their final positions. 


\section{Conclusions}

The feasibility study of an under-actuated parallel manipulator with 6-PUS topology, destined to handle work-tables in CNC machine tools, has been presented. The proposed device exploits the fact that, in such an application, the path between the initial and final poses of the mobile platform is not assigned to reduce the number of actuators to only one. For the proposed manipulator, all the hardware critical points have been addressed. Its kinematic and static characteristics have been discussed, and the availability of the solutions to all the problems involved in its design and control has been verified. Two motion strategies that can be used in the path-planning algorithms have been proposed.

A formalization of the allowed rearrangements using group theory will probably provide a deeper insight into this path planning problem (Joyner, 2002). This is certainly a point that deserves further attention

\section{References}

Bernier, D., Castelain, J.-M., and Li, X. (1995). A new parallel structure with six degrees of freedom. In Proceedings of the 9th World Congress on the Theory of Machines and Mechanisms, Milan (Italy), 8 - 12.

Boye, T., and Pritschow, G. (2005). New transformation and analysis of a N-DOF LINAPOD with six struts for higher accuracy. Robotica 23: 555-560.

Di Gregorio, R. (2002). Singularity-locus expression of a class of parallel mechanisms. Robotica 20: 323-328.

Honegger, M., Codourey, A., and Burdet, E. (1997). Adaptive control of the Hexaglide a 6 dof parallel manipulator. In Proc. of the 1997 IEEE Int. Conf. on Robotics and Automation, Albuquerque, 543-548.

Husty, M.L. (1996). An algorithm for solving the direct kinematics of the Stewart-Gough platform. Mechanism and Machine Theory 31(4): 365-380.

Innocenti, C. (2001). Forward kinematics in polynomial form of the general Stewart platform. Journal of Mechanical Design 123(2): 254-260.

McCarthy, J.M. (2000). Geometric Design of Linkages. Springer-Verlag, New York, chapter 12: 272-283.

Merlet, J.-P. (1989). Singular configurations of parallel manipulators and Grassmann geometry. Int. J. of Robotics Research 8(5): 45-56.

Merlet, J.-P. (2006). Parallel Robots. Dordrecht, The Netherlands: Springer, $2^{\text {nd }}$ edition.

Pritschow, G., Eppler, C., and Lehner, W.-D. (2002). Highly dynamic drives for parallel kinematic machines with constant arm length. In Proc. 1st Int. Colloq., Collaborative Res. Center, Vol. 562, Braunschweig, Germany, 199-211.

St-Onge, B.M., and Gosselin, C.M. (2000). Singularity analysis and representation of the general Gough-Stewart platform. Int. J. of Robotics Research 19(3): 271-288.

Joyner, D. (2002). Adventures in Group Theory. Rubik's cube, Merlin's machine and other mathematical toys. The Johns Hopkins University Press, Baltimore (USA). 